\title{
Near-Infrared Fiber Imager for the VLTI
}

Ralph Neuhäuser ${ }^{1}$, Andreas Tünnermann ${ }^{2}$, Marc Hempel ${ }^{1}$, Bringfried Stecklum $^{3}$, Jena-Peter Ruske ${ }^{4}$, Eike Guenther ${ }^{3}$, Artie Hatzes ${ }^{3}$, Rolf Chini ${ }^{5}$, Roland Lemke ${ }^{5}$, Günther Wuchterl ${ }^{1}$, and Oskar von der Lühe ${ }^{6}$

1 Astrophysikalisches Institut und Universitäts-Sternwarte, Schillergäßchen 2, D-07745 Jena, Germany rne@astro.uni-jena.de

2 Institute for Applied Physics, Universität Jena, D-07745 Jena, Germany

3 Thüringer Landessternwarte Tautenburg, D-07778 Tautenburg, Germany

4 GC-Tec, D-07745 Jena, Germany

5 Ruhr-Universität Bochum, Astronomisches Institut, Universitätsstr. 150, D-44780 Bochum, Germany

6 Kiepenheuer-Institut für Sonnenphysik, Schöneckstr. 6, D-79104 Freiburg, Germany

\begin{abstract}
.
For detection of massive close-in exo-planets around other stars, a contrast of $\sim 0.3 \%$ is sufficient and can be achieved with state-of-the-art single-mode fibers as spatial filter. For lower-mass planets, much higher accuracy is neccessary. Apart from the atmosphere, there are several sources of noise which degrade the phase stability of interferometers based on bulk optics by changes in the optical path. For direct detection of low-mass exo-planets and for direct observation of convection on giant stars, our two main science goals, as well as for secondary science goals such as lower-mass faint circumstellar disks around both nearby low-mass stars as well as more distant high-mass stars and for the direct detection of close spectroscopic binary ( $\mathrm{T}$ Tauri) stars and to follow their orbits for direct dynamical mass determination, we need more than two apertures to fill the uv-plane, again very complicated with bulk-optics. Integrated optics allows to solve these problems and to minimize the components. We have experiences in the development and fabrication of integrated-optical circuits and modulators in various wavelength ranges, i.e. from visible to the telecommunication band, as well as integrated-optical sensor systems like interferometers. Starting from two channel interferometers for the J-band at $1.55 \mu \mathrm{m}$ based on Lithiumniobat, we will develop multichannel-interferometer systems for longer wavelengths. Such systems with new materials and fibers for the near- and thermal infrared can then be used, e.g., for 2nd Gen VLTI instrumentation.
\end{abstract}

\section{Introduction}

We propose to build a Near-Infrared Fiber Imager (NIFI) based on integrated optics for the Very Large Telescope (VLT) Interferometer (VLTI) at the European Southern Observatory (ESO) on Cerro Paranal, Chile. 
As detailed in the next section, we need to achieve stable fringes for long integration times with visibilities going down to $10^{-4}$, which is not yet achieved with current-generation instrumentation. This is partly due to noise inherent in bulk-optics. Hence, we suggest to build a new instrument based completely on integrated optics, i.e. fibers from the telescope feed to the interferometric light combination and onwards to the detectors.

We will first demonstrate this technique in the J- and/or H-band and will then adapt it to the K-band and possibly to the thermal IR later. We plan to combine one to three UTs plus ATs.

\section{Science case(s)}

We propose to build an instrument which is open to the whole astrophysical community and offers science cases in various fields of Galactic and Extragalactic astronomy. Our own design driver for NIFI are long-term stability for very low visibilities to detect young hot low-mass extra-solar planets close-in to their host stars and convection on the surface of giant stars.

Our NIFI instrument will overcome stability problems known from bulk optics interferometers of the first generation. The topic of planet formation and direct observations of extrasolar planets is of large interest in modern astrophysics, in line with the origins theme, and also in connection with astro-biology, the ultimate question of life on other planets.

\subsection{Extrasolar Planets and their Environments}

The formation processes of stars and planets are one of the most important parts of astrophysical research. All extrasolar planets detected so far are Jupiter-like objects in very close orbits, discovered by the radial velocity technique, which gives a lower mass limit on the companions (hence, planet candidates). In addition, there are a very few planets (or candidates) detected directly, including GQ Lup b discovered by us (Neuhäuser et al. 2005) with Adaptive Optics Imaging at the ESO VLT; for such a planet detected directly, one cannot determine the mass directly by Keplers law, because the orbital period is too long (1000 yrs). Hence, neither for radial velocity planet candidates (indirect detection, close orbit) nor for direct detections (wide orbits) one can determine the mass directly, so that the planet nature remains somewhat uncertain. To overcome the limitations of resolution by conventional methods of planet detection like radial velocity surveys and direct imaging and to study close-in companions and earth-like planets, it is necessary to carry out interferometric observations. Our scientific goals are direct interferometric observations of extrasolar planets as well as the visual detection (resolving) of close binary stars of the $\mathrm{T}$ Tauri type - for direct mass determination, in order to test and calibrate theoretical evolutionary tracks and isochrones - partly developed by us. For the test and calibration 
of such theoretical models, convection need to be understood much better than so far; hence, we plan to observe convection directly on the surface of giants with NIFI. Furthermore, we will study massive young stars, analyse in detail the structure of circumstellar disks of nearby young late-type stars, where planets form.

Up to now more than 100 extrasolar planets have been detected indirectly, nearly all of them by radial velocity variations of their host stars. Since the inclination of the orbit remains unknown, these indirect detections only reveal the minimum masses and therefore such objects can only be considered as candidates. In the rare case of a transit the inclination, mass, radius, and the density of the transiting object can be derived and thus planets can be confirmed. Extrasolar planets can be divided into two categories: shortperiod planets with circular orbits and long period planets often on eccentric orbits. Among almost 150 extrasolar planets known to date, one sixth has periods with less than 10 days. The radial velocity technique is only capable of detecting close in planets since the gravitational pull on the host star is too weak for wider orbits. Another method to detect planets on orbits between 2 and $10 \mathrm{AU}$ is via astrometry, i.e., the wobbling of the host star in both right ascension and declination. Furthermore, it is possible to detect extrasolar planets by direct imaging, which is very challenging due to the large brightness difference between star and planet. In the case of young planets this method is applicable because young planets still contract and thus are more easily detectable in the IR spectral range. This method has been applied successfully in the case of GQ Lup by our group (Neuhäuser et al. 2005): the 1 Myr young classical T Tauri star GQ Lup has a L2 companion, comparison with evolutionary models and model atmospheres suggest that the companion - GQ Lup b - has a mass between 1 to 3 Jupiter masses, implying that GQ Lup b is in fact a planet.

The direct detection of extrasolar planets is of major importance to significantly improve our understanding of the formation process of star and planet formation. Therefore it is important also to detect and analyse planets on close-in orbits. Furthermore, circumstellar disks have to be studied. Our group is currently studying the gas content and the dynamics of wellknown circumstellar disks (Hempel \& Schmitt 2003, Hempel et al. 2005) important ingredients to estimate disk masses.

Unfortunately, conventional methods such as radial velocity searches for planets and spectroscopic surveys of circumstellar disks set a limit to the resolution of the observations. A completely different and very powerful method to characterize planetary systems is interferometry: a system consisting of two separate sources (i.e., star \& planet) results in characteristic variations of the visibilitiy when observed at different baselines. Interferometry is capable of overcoming these limits and allows to study close-in extrasolar planets as well as to resolve the inner structure of circumstellar disks. Such observations are indispensable to develop a coherent picture of the process of planet 
formation. Other than the methods described before, interferometry offers much higher angular resolution and is capable of resolving objects with very large differences in brightness. For extrasolar planet studies, interferometers capable of measuring the fringe contrast with high stability are needed. The instruments currently installed at the interferometers do not offer the precision needed to detect low-mass extrasolar planets - a visibility contrast of 0.0001 is needed for this purpose. This can be achieved for long integration times only using integrated optics. Such devices are commonly used in communication technology; their main advantages over conventional bulk-optics are miniaturization of complex optical setups, high modulation- and transfer rates well in the Gbit/s range, and a much better insensitivity to disturbances leading to outstandingly stable fringes. All detection methods together can detect whole planetary systems, i.e. planets at different separations from the host stars, and only interferometry can determine the masses of the planets directly by measuring their light and detecting their position (orbit).

We are searching for planetary (and brown dwarf) companions around young stars by the direct imaging technique, namely around stars from roughly 1 to 100 million years age, all within 40 to $100 \mathrm{pc}$. Given the typical magnitude of such stars in the infrared and the typical magnitude of 5 Jupiter mass objects at the given age range (using Burrows et al. 1997), we can calculate the magnitude difference (or contrast) between star and planet, hence the visibility contrast to be achieved. For the planet (candidates) known so far, the detection of $\epsilon$ Eri b and $\iota$ Hor b may just be possible, with expected visibility contrast of 0.0001 , but this is roughly the limit of the current instrumentation AMBER, which is actually not yet achieved. The most important part in the target selection is the young age of the primary star and, hence, the companion, too, so that its still contracting and, hence, emitting light itself. However, even if the companions cannot be detected by interferometry, given their minimum masses, we can certainly find upper mass limits from non-detections, which will be very useful in determining their true masses and their nature (below 13 Jupiter mass, then planet; or above 13 Jup mass, then brown dwarf).

\subsection{K-Giant Surfaces}

Due to their large surfaces, K giant single stars are perfectly suitable for VLTI measurements. Indeed, $\mathrm{K}$ giants are known to show surface structures: Choi et al. (1995) found CaII variability with periods between $60-160$ days in 10

out of $12 \mathrm{~K}$ giant stars. This can be interpreted as rotational periods which are indirectly visible by means of CaII variability studies. Many K giants are also known to show variable radial velocities: Hatzes \& Cochran (1993) found periods between 233 - 643 days in $3 \mathrm{~K}$ giant stars. Furthermore, Setiawan et al. (2004) found a long-term variability of the K giant HD 78647 correlated with variability in the line profiles.

To explain the observed variability of the $\mathrm{K}$ giant $\alpha$ Tau by means of stellar 
surface structures, roughly one third of the stellar surface would have to be covered with starspots resulting from magnetic activity. The angular diameter of 21 mas allows us to resolve such a starspot on $\alpha$ Tau. Planets as companions may also be responsible for long-term variability (Frink et al. 2002, Setiawan et al. 2003). The VLTI will be capable of testing this hypothesis, i.e., to detect planets via astrometric measurements. The expected astrometric signal is of the order of $\sim 0.3$ mas. Thus it is not detectable using one VLT UT alone, but it is feasible to resolve this with the VLTI. For the current instrumentation of first generation instruments at the VLTI only a very limited number of K giants is detectable. Our proposed instrument allows to detect far more such objects. Thus it will be possible to carry out statistical studies of K giant surfaces: up to now $135 \mathrm{~K}$-type stars - almost all of them giants - are known to have an angular diameter above 5 mas making them potential targets for such a study. Our group (Setiawan \& Hatzes) have carried out a survey of about $80 \mathrm{~K}$ giants studying variability and radial velocities. Thus we have a representative subsample of interesting objects.

\section{Technology: Integrated Optics}

The direct detection of extrasolar planets is based on sophisticated brightness measurements with respect to the enormous intensity ratio between central star and planet. With interferometric telescope systems like ESO-VLTI and Keck Interferometer it is possible to synthesise apertures, which have a substantial narrower PSF compared to single telescopes. For young warm exoplanets $(1000 \ldots 1500 \mathrm{~K})$ the intensity ratio between star and planet amounts to approximately $3 \cdot 10^{-5}$ to $3 \cdot 10^{-4}$ in the near infrared. They contract significantly and gain gravitational energy, which is emitted in the infrared leading to by some orders of magnitude higher brightness compared to old planets (i.e. Burrows et al. 1997, Wuchterl \& Tscharnuter 2003). Consequently, an interferometer for investigation of extrasolar planets must allow to measure the contrast of an interference pattern with high accuracy, which implies a high phase stability. Simulations show, that the contrast error should be lower than $0.3 \%$ for interferometric verification of planets similar to $51 \mathrm{Pe}-$ gasi (de Foresto et al. 1997). In practise such precision can be reached using singlemode optical fibres as spatial filters (Perrin et al. 1997). But for the verification of lower-mass exoplanets a considerably higher precision is necessary.

There are some factors influencing the phase stability of bulk-optics interferometers besides atmospheric turbulences which lead to fluctuations and systematic changes of the optical path length, i.e., seismic activity and temperature gradients. At the Institute of Applied Physics of the University of Jena (IAP) we have carried out investigations to spatial filtering using singlemode fibres (Zeitner et al. 1999). 
Since the investigation of the trajectory of exoplanets (and thus also its mass) is possible with relatively good accuracy using two apertures, for the direct imaging a combination of more apertures, that is a covering in the uv-plane is needed as good as possible. In the case of VLTI (four $8.2 \mathrm{~m}$ and some $1.8 \mathrm{~m}$ telescopes) maximum of 21 baselines are possible where interferometric signals can be measured. Optical designs for the combination of some apertures basing on bulk optics are extremely difficult and thus susceptible to systematic and random perturbations which influence the phase stability.

The astronomic precision in the range of microarcseconds, for which a reference object is needed (dual feed) will be increased by the improvement of the phase stability using the proposed integrated-optical concept. Integrated optics allows a considerable miniaturisation of complex optical systems. Especially the combination of integrated-optical circuits with optical fibres forms a completely atmospherically protected light path leading to substantial stability increase of the system.

In optical telecommunications integrated-optical components are used for fast light modulation, splitting and combining as well as multiplexing of data channels. They are fabricated in large quantities. Optical glasses, the ferroelectric crystal lithium niobate, compound semiconductors and silicon are used as substrate materials mostly. The waveguides are fabricated by micropatterning, ion exchange or metal indiffusion or by stacked deposition of semiconducting or oxidic layers with different composition followed by microfabrication.

The advantages of integrated-optical systems against bulk-optics are the small size, stability, very high data rates up to the $40 \mathrm{Gbit} / \mathrm{s}$ range, good reproducible interferometric characteristics and extinctions as well as the proof against outer influences to the optical path. Interferometric principles are used in modulators, switches, wavelength multiplexers and filters (Murphy 1999). For example an amplitude modulator is implemented as a two beam interferometer, where the optical path length can be controlled by means of electrooptics. For this purpose an electrode system is positioned in plane of a Mach-Zehnder-waveguide structure. The modulator transmission follows a quadratic cosine function if a voltage is applied.

Integrated-optical devices are available almost only for telecommunication purposes, where standard devices for wavelengths around the $1.3 \mu \mathrm{m}$ and $1.55 \mu \mathrm{m}$ wavelength are used. Other elements are not available except modulators for the $800 \mathrm{~nm}$ and $1060 \mathrm{~nm}$ range. In research institutes like the IAP waveguides and devices for a large number of wavelengths and basing on various substrates are investigated including also photonic crystal waveguides and fibres.

There exists a variety of possibilities to imply passive integrated-optical elements into interferometers for astronomic purposes. Especially two or more telescopes can be networked to interferometers with large base length. In Malbet 1999 interferometric schemes are presented in form of block diagrams. A 
part of these assemblies can be implemented as integrated or fibre optics advantageously. Successful experiments have been done using commercial passive structures in laboratory (Berger 1999). The up to now highest integration was realised in form of the combination of eight inputs using an integrated-optical interferometric structure by Berger 2000. A disadvantage is the relatively low spectral bandwidth.

For control of interference it is advantageous to combine more functions in the integrated interferometer. Especially the implementation of phase shifters allows an increase of measurement accuracy. These elements have not been investigated for astronomic purposes in the past and have been realised by mechanical means.

A problem is the efficient incoupling of stellar light into optical fibres. The technical possibilities and limits have been described by Shaklan and Roddier (1999) in theoretical way. However, experiments have been done mainly in laboratory using lasers and discharge lamps.

There are great experiences in the field of integrated-optical elements especially in glass and electrooptic materials like lithium niobate LiNbO3 and potassium titanyl phosphate (KTP). Diverse elements like interferometers for sensors, polarisers as well as electro-optic phase and amplitude modulators for wavelengths between $450 \mathrm{~nm}$ and $1550 \mathrm{~nm}$ have been realised (Ruske et al. 1995b, Ruske et al. 1999). Waveguides in KTP have an outstanding position in this field. Beside the electrooptic function they are able to guide light of a comparably large spectral bandwidth in singlemode (Rottschalk et al. 1997). This is the prerequisite for broadband electrooptical controllable interferometric devices for light modulation and colour mixing. The optical bandwidth amounts to be already $200 \mathrm{~nm}$ in the visible and much more in the infrared. These waveguides and modulators have been developed for colour image generation.

The (i) theoretical modelling and experimental verification of waveguides in single- and multimode technique with respect to operation ranges and guiding parameters for application in beam combination, beam splitting and modulation (Rottschalk et al. 1995, 1997), the (ii) concept of integratedoptical modulators for colour image generation with laser light in form of a power proofed and broadband solution (Ruske et al. 1995a, Ruske et al. 1999) and (iii) the fabrication of demonstrators for colour image generation systems (Ruske et al. 1998, Ruske et al. 1999) have been focal points in these projects. Interferometric modulators with contrast ratio of about 500:1 in the visible and 5000:1 in the infrared for modulation frequency of more than some $100 \mathrm{MHz}$ and guided optical power up to $100 \mathrm{~mW}$ have been realized in reproducible way. These devices will find application in small displays and apparatuses for printing and photofinishing. Devices for applications in the near infrared (for example $830 \mathrm{~nm}, 1060 \mathrm{~nm}, 1550 \mathrm{~nm}$ ) have been successful realized by means of industry cooperation. 
Therefore it was possible for the first time to fabricate singlemode waveguides for the visible range and to show a precise amplitude modulation with high extinction using magnesium doped lithium niobate. These modulators can be used in laser based photofinishing systems, which are currently equipped with complex bulk optics.

The fabrication of waveguides and modulators for ultrashort pulses in the range of one picosecond and peak power of more than 120 Watts is possible, which is an international outstanding value. Such ultrashort pulse laser systems for use in micro material processing and biotechnology can be configured more efficient and simple (Ruske et al. 2003a,b).

Further increase of the guidable power in singlemode waveguides was successful by change of the waveguide geometriy. A special property of KTP allows an increase of the waveguide cross section thus causing a decrease of the optical power density in the waveguide. These waveguide requires a new modulation concept, which was demonstrated as asymmetric diffraction amplitude modulator. So it is possible to guide and modulate light of some watts in singlemode (Werner et al. 2003). Further investigations are due to material improvement for integrated-optical purposes, especially using upgrading of lithium niobate substrates by liquid phase epitaxial layer deposition. An important point are the activities concerning the use of integrated-optical devices in aerospace and astronomy. So flight proofed high-power gigahertz phase modulators have been realized for optical communication between geostationary and low earth orbit satellites. Its operation wavelength is $1064 \mathrm{~nm}$, the transmitted power is in the range of 1 Watt that is extremely high for integrated devices. These devices have been realised for the first time (Ruske et al. 2003a).

To open the mid-infrared the use of photonic crystals and photonic crystal fibres is advantageous. Its function bases on the combination of allowed and forbidden zones for light transmission due to Bragg reflection on microstructures in planar as well as fibre geometry. Beside the increase of the IR transmission range it is possible to control the dispersion and to enlarge the guidable power in optical fibres. In planar structures it is possible to connect angled waveguides without bends thus enlarging the package density of integrated-optical circuits. The waveguides are prepared by microfabrication technique. They consist of a periodic sequence of high and low refracting structures in sub wavelength geometry, mostly as hexagonal sequence of holes in the substrate. By suitable arrangement of allowed and forbidden zones it is possible to force the light onto a predetermined path. Some work concentrates on photonic crystal waveguides with high index contrast mostly on the basis of semiconductors, for example GaAs against $\mathrm{Al} 2 \mathrm{O} 3$ or air (Chow et al. 2001, Olivier et al. 2001). Other groups work with low index contrast in high refracting materials, for example GaAs against Ga1-xAlxAs (Bogaerts et al. 2001, Lalanne 2002). However the coupling to optical fibres is difficult due to 
the high index of refraction of the semiconductors. The losses are relatively high and amount up to $30 \mathrm{~dB} / \mathrm{mm}$.

Therefore, we favour photonic crystal waveguides with low index contrast (Tünnermann et al. 2004). Here a layer system is used which consists of a $\mathrm{Nb2O} 5$ layer on thermal oxidised silica. A cladding layer is formed by sputtered silica. So a symmetric layer waveguide is obtained, which guides the light in vertical direction. The lateral confinement is done by a photonic crystal structure made of $370 \mathrm{~nm}$ diameter holes with a depth of $1.1 \mu \mathrm{m}$ hexagonal arranged with a period of $595 \mathrm{~nm}$. It has been realised by a combination of electron beam lithography, reactive ion beam etching (RIE) and inductive coupled plasma etching (ICP). So singlemode waveguiding with an attenuation of $1.7 \mathrm{~dB} / \mathrm{mm}$ was obtained. This relatively high value has to be associated with the some tenth millimetres short structures necessary for waveguide bending since devices are realised by small photonic structures combined with straight conventional low loss rib waveguides. These photonic structures are a future oriented possibility to increase the package density of integrated-optical devices. However, their fabrication is very difficult and can only be realised with the expensive electron beam lithography. The structures are two dimensional now, but stackable in future. With photonic structures the middle infrared can be opened up for integrated optics.

\section{Summary}

Infrared interferometry with integrated optics is an innovative method in extrasolar planet studies and necessary to obtain new insight of close-in companions. This is indispensable to solve long-standing questions concerning planet-formation, i.e., mass-distributions, metallicities, orbits, and atmospheres of extrasolar planets and brown dwarfs.

Direct detection and interferometric observations of extrasolar planets are important goals of european astrophysics, as stated by ESO, ESA, and EU panel long-term strategies. Around 2015, the DARWIN mission will be launched by the European Space Agency. The challenging main goal of this mission is to search for Earth-like planets and possible signs of life on extrasolar planets with a spaceborn interferometer. To do this in an efficient way, possible targets have to be detected. In the course of our proposed project we will be able to determine parameters of extrasolar planets and planetary systems with high accuracy and thus be able to provide a list of candidates for planets harbouring extrasolar life.

\section{References}

1. Berger J.-P., K. Rousselet-Perraut, P. Kern, F. Malbet, I. Schanen-Duport, F. Reynaud, P. Haguenauer, P. Benech, 1999, Astron. Astrophys. Supp. Ser. 139, S. 173 
2. Berger J.-P., P. Benech, I. Schanen, G. Maury, F. Malbet, F. Reynaud, 2000, Proceedings SPIE, Vol. 4006, S. 986

3. Bogaerts W., Bienstman P., Taillaert D., Baets R., DeZutter D., 2001, IEEE Phot. Technol. Lett. 13, S. 565

4. Burrows A., Marley M., Hubbard W. et al. 1997, ApJ 491, 856

5. Choi, H.-J., Soon, W., Donahue, R.A., Baliunas, S.L., Henry, G.W., 1995, PASP 107,744

6. Chow E., Lin S.Y., Wendt J.R., Johnson S.G., Joannopoulos J.D., 2001, Optics Letters 26, S. 286

7. de Foresto C.V., J.-M. Mariotti, G. Perrin, 1997, in: Science with the VLT Interferometer, F. Paresce, Springer Verlag 1997, S. 86

8. Frink, S., Mitchell, D.S., Quirrenbach, A., Fischer, D.A., Marcy, G.W., Butler, R.P. 2002 ApJ 576, 478

9. Hatzes, A.P., Cochran, W.D. 1993 ApJ 413, 339

10. Hempel, M., Schmitt, J.H.M.M. 2003, A\&A, 408, 971

11. Hempel, M., Robrade, J, Ness, J.-U., Schmitt, J.H.M.M. 2005, A\&A, accepted

12. Lalanne P., 2002, IEEE J. Quantum Electron. QE-38, S. 800

13. Malbet F., P. Kern, I. Schanen-Duport, J.-P. Berger, K. Rousselet-Perraut, P. Benech, 1999, Astron. Astrophys. Supp. Ser. 138, S. 135

14. Murphy E.J., 1999, Integrated optical circuits and components, Marcel Dekker

15. Neuhäuser R., Guenther E.W., Wuchterl G., Mugrauer M., Bedalov A., Hauschildt P., 2005, A\&A 435, L13

16. Olivier S., Rattier M., Benisty H., Weisbuch C., Smith C.J.M., DeLaRue R.M., Krauss T.F., Oesterle U., Houdre R., 2001, Phys. Rev. B 63, Artikel Nr. 113311

17. Perrin G., V. C. de Foresto, S.T. Ridgway, J.-M. Mariotti, N.P. Carleton, W. Traub, 1997, in: Science with the VLT Interferometer, F. Paresce, Springer Verlag 1997, S. 318

18. Ruske J.-P., M. Rottschalk und B. Unterschütz, 1995b, Proceedings of the 7th European Conference on Integrated Optics ECIO 95, Delft, Niederlande, 03.-06. April 1995, S. 383

19. Ruske J.-P., B. Zeitner, W. Biehlig, E. Werner, A. Tünnermann, 1999, LaserOpto 31 (2), S. 40

20. Ruske J.-P., M. Rottschalk, B. Zeitner, V. Gröber, A. Rasch, 1998, Electronics Letters 34 (4), S. 363

21. Ruske J.-P., Zeitner B., Tünnermann A., Rasch A.S., 2003a, Electron. Lett. 39, 1048

22. Ruske J.-P., Werner E.A., Zeitner B., Tünnermann A., 2003b, Electron. Lett. 39, 1442

23. Rottschalk M., J.-P. Ruske, St. Steinberg, K. Hornig, G. Hagner und A. Rasch, 1995, Journal of Lightwave Technology 13 (10), S. 2041

24. Rottschalk M., J.-P. Ruske, B. Unterschütz, A. Rasch, V. Gröber, 1997, J. Appl. Phys. 81 (6), S. 2504

25. Setiawan, J., Hatzes, A.P., von der Lhe, O., Pasquini, L., Naef, D., da Silva, L., Udry, S., Queloz, D., Girardi, L. 2003 A\&A 398, L19

26. Setiawan, J., Pasquini, L., da Silva, L., Hatzes, A.P., von der Lhe, O., Girardi, L., de Medeiros, J.R., Guenther, E. 2004 A\&A 421, 241

27. Shaklan S., F. Roddier, 1988, Appl. Opt. 27 (11), S. 2334

28. Tünnermann A., Schreiber T., Augustin M., Limbert J., Will M., Nolte S., Zellmer H., Iliew R., Peschel U., Lederer F., 2004, Photonic crystals in ultrafast 
optics, In: Advances in Solid States Physics, B. Kramer (Ed.) Vol. 44, 117 Springer Berlin

29. Werner E.A., Ruske J.-P., Zeitner B., Biehlig W., Tünnermann A., 2003, Opt. Commun. 221, 9

30. Wuchterl, G., Tscharnuter, W.M., 2003 A\&A 398, 1081

31. Zeitner B., Ruske J.-P., Werner E., Rottschalk M., Rasch A., Tünnermann A., 1999, Abschlussbericht BMBF-Verbundprojekt FABIAN (FKZ 16 SV 404/9, Laufzeit: 01.01.1996 - 31.07.1999): Integriert-optischer Farbmischer für ein Laserfarbbilderzeugungssystem 